\title{
Daytime Napping and Nighttime Sleep During Pregnancy and Preterm Birth in Iran
}

\author{
Farnaz Shaliha', Maryam Mozaffari', Faeze Ramezani ${ }^{1}$, Hamideh Hajnasiri' ${ }^{2}$, Farnoosh Moafi \\ ${ }^{1}$ Student Research Committee, Qazvin University of Medical Sciences, Qazvin, Iran; ${ }^{2}$ Social Determinants of Health Research Center, Research \\ Institute for Prevention of Non-Communicable Diseases, Qazvin University of Medical Sciences, Qazvin, Iran
}

Objectives: This study investigated the relationship between sleep quality during pregnancy and preterm birth.

Methods: This longitudinal study was conducted between August 2018 and May 2019. The participants were 150 pregnant women who had been referred to 7 healthcare centers in the city of Qazvin, Iran and met the inclusion criteria. The Petersburg Sleep Quality Index, the Epworth Sleepiness Scale, and 2 questions about daytime sleep status and a demographic questionnaire were administered at 14-18 weeks and 28-32 weeks of gestation. Data were analyzed using the Mann-Whitney test, the Fisher exact test, and univariate and multivariable logistic regression.

Results: In the present study, poor sleep quality affected $84.7 \%$ of the participants at $14-18$ weeks and $93.3 \%$ at $28-32$ weeks of gestation. The final model for preterm birth prediction incorporated age and the Petersburg Sleep Quality Index score in the second and third trimesters. Preterm birth increased by $14 \%$ with each unit increase in age. With each unit increase in the Petersburg Sleep Quality Index score in the second and third trimesters, preterm birth increased by $42 \%$ and $28 \%$, respectively, but the $p$-values of these factors were not significant.

Conclusions: Although a significant percentage of pregnant women had poor sleep quality, no significant relationship was found between sleep quality during pregnancy and preterm birth.

Key words: Pregnancy, Premature birth, Sleep, Iran

\section{INTRODUCTION}

Preterm birth, which is defined as prenatal birth before 37 weeks of gestation, is the leading cause of infant mortality and is associated with many complications [1]. Each year, approxi-

Received: August 1, 2020 Accepted: March 11, 2021

Corresponding author: Farnoosh Moafi

Social Determinants of Health Research Center, Research Institute for Prevention of Non-Communicable Diseases, Qazvin University of

Medical Sciences, P.O. Box 34199-15315, Qazvin, Iran

E-mail: f.moafi.sbmu@gmail.com

This is an Open Access article distributed under the terms of the Creative Commons Attribution Non-Commercial License (https://creativecommons.org/licenses/by$\mathrm{nc} / 4.0 /$ ) which permits unrestricted non-commercial use, distribution, and reproduction in any medium, provided the original work is properly cited. mately 15 million babies are born prematurely worldwide [2]. The preterm birth rate in the United States has been on the rise since 2014, reaching $9.93 \%$ of births in 2017 [3]. In developing countries, especially in South Asia and sub-Saharan Africa, the preterm birth rate is estimated to be more than $15 \%$ [4]. Approximately $90 \%$ of preterm deliveries occur in developing countries, of which $85 \%$ are reported in Asia and Africa [5]. The preterm birth rate in Iran is estimated to be between $5.40 \%$ and $19.85 \%$, indicating that preterm birth is a relatively common problem [6].

The etiological, epidemiological, and clinical nature of preterm birth in many cases is unidentified and idiopathic. Researchers have identified preterm birth as a multifactorial complication and have attributed it to individual, social, environ- 
mental, psychological, medical, genetic, biological, and infertility-related factors $[7,8]$. Numerous studies have been conducted in this field, and factors reported to affect preterm birth include infection, premature rupture of membranes, history of preterm birth, multiple births, pre-eclampsia, use of assisted reproductive techniques, pregnancy at an early age, low education, inadequate care during pregnancy, obesity, stress, gestational diabetes, placenta previa, and reproductive system disorders [8-10]. Given that the cause of two-thirds of preterm births remains unknown, it seems that the best way to prevent this complication is to identify pregnant women with risk factors and to seek out risk factors among biological and psychosocial causes [11].

Pregnancy is a special period in life that is associated with significant changes in sleep patterns and sleep quality. It can result in sleep disorders such as insomnia, snoring, nocturnal awakening, restless legs syndrome, and obstructive sleep apnea during the day [12-14]. The prevalence of these disorders has been estimated to be $58 \%$ in the second trimester of pregnancy and $66 \%$ in the third trimester [15]. Sleep quality during pregnancy and postpartum is affected by hormonal and physiological changes. Sex hormones and gonadotropins may also contribute to sleep disorders [16]. Frequent urination, inability to find a comfortable position, pelvic pain, leg cramps, back pain, hunger, and itchy skin are some of the factors that affect the sleep quality of pregnant women [17]. Sleep disorders significantly affect the quality of life and reduce daily physical, psychological, and social functioning [18-20], and can also cause neuroendocrine, metabolic, and inflammatory changes [21]. Sleep deprivation and sleep disorders during pregnancy may increase rates of pre-eclampsia, gestational diabetes, anxiety, postpartum depression, reduced pain tolerance, prolonged labor, and cesarean delivery [22-24].

One of the undesirable consequences of poor sleep quality during pregnancy may be preterm birth [25]. In this regard, studies have reported contradictory results. Some studies have found a positive association between poor sleep quality and preterm birth [25-28], but this finding was not reported in other studies $[29,30]$. These studies have also been criticized; for example, the quantity and quality of sleep during pregnancy were examined by asking only 2 questions [27]. In another studies $[25,29,30]$, sleep disorders were examined only in the third trimester. Most of these studies also investigated maternal sleep during the night and did not examine the relationship between maternal daytime sleep and preterm birth. There- fore, due to the high prevalence of sleep disorders during pregnancy, and considering the contradictory information about the relationship between poor sleep quality and preterm birth and the importance of the effects of preterm birth on infant health, this study was conducted to determine the relationship between sleep quality during pregnancy and preterm birth.

\section{METHODS}

\section{Study Design and Population}

In this longitudinal study, multi-stage sampling was used, and the study population was pregnant women referred to healthcare centers in Qazvin, Iran, between August 2018 and May 2019. The cluster random sampling method was used in this study. Quotas were allocated to each of the 7 healthcare centers in Qazvin according to the number of pregnant women referred to each center within 1 month before sampling. The samples were selected using convenience sampling and they entered the study based on the inclusion criteria. The inclusion criteria were being Iranian; being able to read and write; not consuming alcohol, drugs, or tobacco; being 18-35 years old; being at 14-18 weeks of gestation; being a primigravida, having a low-risk and singleton pregnancy; and not having any chronic diseases or history of a mental illness (according to the patient and based on the patient's records). In this study, according to the findings of other studies [31], the ratio of $p$-val$\mathrm{ue}=0.5$ was considered to find the sample size, and by using $\alpha=0.05, Z_{1-\alpha / 2}=1.96$, and $d=0.05$, the minimum sample size was estimated at 150 .

\section{Instruments}

Data collection tools included a demographic questionnaire, the Petersburg Sleep Quality Index (PSQI), and the Epworth Sleepiness Scale (ESS). In addition, daytime sleep status was examined with 2 questions: "Do you take a nap during the day?" and "How long do you nap during the day?"

The PSQI is a self-rated questionnaire that evaluates sleep quality. The reported sensitivity and specificity of the PSQI are $89.6 \%$ and $86.5 \%$, respectively, for identifying sleep disorders in adults. The index includes 7 items: subjective sleep quality, sleep latency, sleep duration, habitual sleep efficiency, sleep disturbances, use of sleeping medication, and daytime dysfunction. Each item is scored on a scale from 0 to 3 using a 4-point Likert scale. The total score is $0-21$, and a total score of 
5 or higher is indicative of poor sleep quality. The reliability of the Iranian version of the PSQI has been examined and the Cronbach's alpha has been reported to be 0.79 [32].

The ESS is a self-administered questionnaire measuring sleepiness during 8 common situations in daytime life. The range of the score for each item is $0-4$ and the total score is $32-42$. A score of 11 or higher is indicative of poor daily sleep quality. Cronbach's alpha of this questionnaire was reported to be 0.77 for the population of Iranian pregnant women [29].

\section{Procedure}

Data collection was performed in 3 stages after obtaining the necessary permissions. In the first stage, at 14-18 weeks of gestation, the demographic questionnaires, the PSQI, the ESS, and the daytime sleep status questions were completed. In the second stage, at the 28-32 weeks of gestation, except for the demographic questionnaire, the rest of the questionnaires were completed again. In the third stage, after delivery, information about the delivery was collected over the phone. Moreover, the patients' records were also checked to confirm the information that they provided. Preterm birth was defined as less than 37 weeks of gestation based on the last menstrual period or based on the ultrasound measurements taken in the first trimester.

\section{Statistical Analysis}

Data analysis was performed using SPSS version 23 (IBM Corp., Armonk, NY, USA). Descriptive statistics (e.g., frequency and percentage to describe qualitative data and mean and standard deviation to describe quantitative data) were used. The Kolmogorov-Smirnov test was used to assess the normality of the data, and it showed that the quantitative variables of the study were not normally distributed $(p<0.05)$. Therefore, to compare the sleep quality in the second and third trimesters of pregnancy, the Mann-Whitney non-parametric test was used to describe qualitative variables, and the Fisher exact test was used to compare them. The significance level was considered to be $p$-value $<0.05$.

Univariate and multivariable logistic regression methods were used to investigate the relationship between predictive variables and preterm birth. In order to determine the variables affecting preterm birth, each of the demographic and sleep variables was individually investigated in the univariate logistic regression analysis, and variables with a significance level less than 0.2 were entered into the multivariable logistic re- gression model, in which the dependent variable was preterm birth. Independent variables included factors affecting preterm birth that were significant in the univariate logistic regression analysis. These variables were entered into the model using the forward Wald (i.e., stepwise) method.

\section{Ethics Statement}

The present study was approved with the code of IR.QUMS. REC.1396.310 by the Ethics Committee of Qazvin University of Medical Sciences, Iran. Informed consent was obtained from all women participating at the time of sampling. They were also provided with adequate explanations about the aim and the type of the study. They were assured that they could leave the study at any time.

\section{RESULTS}

In this study, 158 pregnant women were invited to participate, of whom 5 were excluded from the study due to pregnancy-related complications and 3 were excluded due to incomplete questionnaires. Data from 150 pregnant women were ultimately analyzed. The response rate in the present study was $94.9 \%$.

Of the 150 pregnant women surveyed, $6(4.0 \%)$ had preterm births and 144 (96.0\%) had term births. The mean gestational age in the second trimester was $16.04 \pm 1.47$ weeks, while in the third trimester, it was $29.91 \pm 1.38$ weeks. The mean age of the pregnant women was $25.81 \pm 4.15$ years. The majority of participants had academic education (72.0\%) and were housewives $(82.0 \%)$. A sufficient income was reported by $80.0 \%$ of participants. The results of the univariate logistic regression analysis are presented in detail in Table 1.

The mean PSQI score in the second trimester of pregnancy was $6.53 \pm 2.17$, and in the third trimester of pregnancy, it was $6.89 \pm 2.12$. The mean ESS score was $12.59 \pm 3.76$ in the second trimester and $12.00 \pm 3.11$ in the third trimester of pregnancy. A total of $80.0 \%$ of pregnant women in the second trimester and $68.7 \%$ in the third trimester of pregnancy reported daytime napping. The mean nap duration in the second trimester was $65.40 \pm 54.30$ minutes, and in the third trimester, it was $43.90 \pm 39.89$ minutes. The results of the univariate logistic regression analysis are presented in detail in Table 1.

Table 2 presents the final model for preterm birth predictors, which included age and the PSQI score in the second and third trimesters. The results showed that as age and the PSQI 
Table 1. The result of univariate logistic regression analysis

\begin{tabular}{|c|c|c|c|c|c|c|}
\hline \multirow{2}{*}{ Variables } & \multirow{2}{*}{ Term } & \multirow{2}{*}{ Preterm } & \multirow{2}{*}{ Total } & \multicolumn{3}{|c|}{ Uunivariate logistic regression analysis } \\
\hline & & & & $p$-value & Standard error & $\boldsymbol{\beta}$ \\
\hline \multicolumn{7}{|l|}{ Ddemographic } \\
\hline \multicolumn{7}{|l|}{ Education } \\
\hline Academic degree & $102(70.8)$ & $6(100)$ & $108(72.0)$ & - & - & - \\
\hline None academic degree & $42(29.2)$ & $0(0.0)$ & $42(28.0)$ & 0.998 & 6201.91 & -18.37 \\
\hline \multicolumn{7}{|l|}{ Occupation } \\
\hline Housewife & $117(81.2)$ & $6(100)$ & $123(82.0)$ & - & - & - \\
\hline Employed & $27(18.7)$ & $0(0.0)$ & $27(18.0)$ & 0.998 & 7735.14 & 18.23 \\
\hline \multicolumn{7}{|l|}{ Family income status } \\
\hline Under sufficient & $16(11.0)$ & $0(0.0)$ & $16(11.0)$ & - & - & - \\
\hline Sufficient & $115(80.0)$ & $5(83.0)$ & $120(80.0)$ & 0.999 & 1004.24 & -18.63 \\
\hline In term of savings & $13(9.0)$ & $1(17.0)$ & $14(9.0)$ & 0.253 & 1.13 & -0.57 \\
\hline $\mathrm{Age}^{1}$ & $25.72 \pm 4.08$ & $28.00 \pm 5.62$ & $25.81 \pm 4.15$ & 0.192 & 0.10 & 0.13 \\
\hline \multicolumn{7}{|l|}{ Sleep status } \\
\hline \multicolumn{7}{|l|}{$14-18 w k$} \\
\hline Petersburg Sleep Quality Index' & $6.44 \pm 2.17$ & $8.67 \pm 0.51$ & $6.53 \pm 2.17$ & 0.023 & 0.18 & 0.41 \\
\hline Epworth Sleepiness Scale & $12.53 \pm 3.75$ & $14.00 \pm 4.09$ & $12.59 \pm 3.76$ & 0.348 & 0.09 & 0.09 \\
\hline Daily nap duration (min) & $66.46 \pm 54.87$ & $40.00 \pm 30.98$ & $65.40 \pm 54.30$ & 0.252 & 0.01 & -0.01 \\
\hline \multicolumn{7}{|l|}{ Daily nap } \\
\hline Yes & $116(80.6)$ & $4(66.7)$ & $120(80.0)$ & - & - & - \\
\hline No & $28(19.4)$ & $2(33.3)$ & $30(20.0)$ & 0.414 & 0.89 & 0.72 \\
\hline \multicolumn{7}{|l|}{$28-32$ wk } \\
\hline Petersburg Sleep Quality Index & $6.81 \pm 2.01$ & $9.00 \pm 3.57$ & $6.89 \pm 2.12$ & 0.019 & 0.17 & 0.39 \\
\hline Epworth Sleepiness Scale & $11.97 \pm 3.10$ & $12.67 \pm 3.61$ & $12.00 \pm 3.11$ & 0.593 & 0.11 & 0.06 \\
\hline Daily nap duration (min) & $43.65 \pm 39.34$ & $50.00 \pm 55.85$ & $43.90 \pm 39.89$ & 0.702 & 0.01 & 0.00 \\
\hline \multicolumn{7}{|l|}{ Daily nap } \\
\hline Yes & $99(68.8)$ & $4(66.7)$ & $103(68.7)$ & - & - & - \\
\hline No & $45(31.3)$ & 2 (33.3) & 47 (31.3) & 0.914 & 0.88 & 0.09 \\
\hline
\end{tabular}

Values are presented as number (\%) or mean \pm standard deviation.

${ }^{1}$ Variables selected to enter into the multivariate regression model.

Table 2. Multivariate regression analysis of preterm birth predictors

\begin{tabular}{|c|c|c|c|c|c|}
\hline Variables & $\boldsymbol{\beta}$ & SE & Wald & $p$-value & OR $(95 \% \mathrm{CI})$ \\
\hline Age & 0.13 & 0.109 & 1.59 & 0.206 & $1.14(0.92,1.42)$ \\
\hline \multicolumn{6}{|l|}{ Petersburg Sleep Quality Index (wk) } \\
\hline $14-18$ & 0.35 & 0.227 & 2.42 & 0.119 & $1.42(0.91,2.22)$ \\
\hline $28-32$ & 0.25 & 0.209 & 1.48 & 0.224 & $1.28(0.85,1.94)$ \\
\hline Hosmer and Lemeshow test: $\chi^{2}=5.95$ & $\mathrm{df}=8$ & & & & \\
\hline \multicolumn{6}{|c|}{ Model summary: -2 log likelihood $=40.827 ;$ Cox-Snell $R^{2}=0.062 ;$ Nagelkerke $R^{2}=0.216$} \\
\hline \multicolumn{6}{|c|}{ Omnibus test of model coefficients: $\chi^{2}=9.55 \quad \mathrm{df}=3 \quad \mathrm{Sig}=0.02$} \\
\hline
\end{tabular}

$\mathrm{SE}$, standard error; OR, odds ratio; $\mathrm{Cl}$, confidence interval; df, degrees of freedom; Sig, significance.

score in the second and third trimesters increased (with a higher score indicating poor sleep quality), the rate of preterm birth increased. The PSQI in the second trimester, with a ratio of 1.42 , was the strongest predictor in the model; however, none of the variables included in the final model were significant. 
In addition, considering the features of the model, based on the Hosmer and Lemeshow test $(p>0.05)$ and the significance of omnibus tests of model coefficients $(p<0.05)$, the final model had good explanatory power and efficiency. This model explained between $0.6 \%$ and $22.0 \%$ of preterm birth variance.

Table 3 depicts the frequency distribution of sleep variables based on the trimester of pregnancy. The results showed that as pregnancy progressed, the PSQI score increased, meaning that sleep quality became worse over the course of pregnancy. Thus, $84.7 \%$ of participants had poor sleep quality in the second trimester and $93.3 \%$ had poor sleep quality in the third trimester, reflecting a significant difference $(p<0.05)$. However, the ESS score decreased in the third trimester of pregnancy compared to that in the second trimester, meaning as the pregnancy progressed, the quality of daytime sleep improved. Thus, $77.3 \%$ of pregnant women in the second trimester and $66.0 \%$

Table 3. Sleep variables across pregnancy

\begin{tabular}{|c|c|c|c|}
\hline \multirow{2}{*}{ Variables } & \multicolumn{2}{|c|}{ Pregnancy (wk) } & \multirow{2}{*}{$p$-value } \\
\hline & $14-18$ & 28-32 & \\
\hline \multicolumn{4}{|c|}{ Petersburg Sleep Quality Index } \\
\hline$<5$ & $23(15.3)$ & $10(6.7)$ & 0.031 \\
\hline$\geq 5$ & $127(84.7)$ & $140(93.3)$ & \\
\hline \multicolumn{4}{|c|}{ Epworth Sleepiness Scale } \\
\hline$<11$ & $34(22.7)$ & $51(34.0)$ & 0.010 \\
\hline$\geq 11$ & $116(77.3)$ & $99(66.0)$ & \\
\hline \multicolumn{4}{|l|}{ Daily nap } \\
\hline Yes & $120(80.0)$ & $103(68.7)$ & 0.021 \\
\hline No & $30(20.0)$ & 47 (31.3) & \\
\hline Daily nap duration & $54.30 \pm 65.40$ & $39.89 \pm 43.90$ & 0.001 \\
\hline
\end{tabular}

Values are presented as number (\%) or mean \pm standard deviation. of those in the third trimester had poor daytime sleep quality $(p=0.01)$. In the second and third trimesters, $80.0 \%$ and $68.7 \%$ of pregnant women reported daytime napping, respectively $(p<0.05)$. The nap duration was $54.30 \pm 65.40$ minutes in the second trimester; and in the third trimester, it was $39.89 \pm$ 43.90 minutes $(p=0.001)$.

As secondary outcomes, data on mode of delivery and birth weight were collected and analyzed in the same way as for preterm birth. In these analyses, preterm birth was examined as a confounding factor in the relationship between sleep variables and mode of delivery and birth weight. In the univariate logistic regression analysis, daytime napping and the ESS score in the second trimester, and nap duration and the PSQI score in the third trimester had a significance level less than 0.2 , so they were entered into the multivariable regression analysis. In the final analysis, daytime napping in the second trimester and the duration of naps in the third trimester significantly predicted the mode of delivery $(p<0.05$, respectively). Thus, if pregnant women nap more during the day in the second trimester and the nap duration is increased in the third trimester, the incidence of cesarean section would be reduced (odds ratio, 0.32 and 0.98 , respectively). The final model for the mode of delivery is presented in Table 4. For birth weight, in the univariate regression analysis, only the PSQI score in the second trimester predicted low birth weight (under $2500 \mathrm{~g}$ ), but the relationship was not significant $(p=0.196)$.

\section{DISCUSSION}

The present study investigated the relationship between sleep quality during pregnancy and preterm birth. Based on the results of the initial data, the risk of preterm birth increased

Table 4. Multivariate regression analysis of cesarean section predictors

\begin{tabular}{|c|c|c|c|c|c|}
\hline Variables & $\beta$ & SE & Wald & $p$-value & OR $(95 \% \mathrm{CI})$ \\
\hline \multicolumn{6}{|l|}{ Sleep status } \\
\hline \multicolumn{6}{|l|}{$14-18$ wk } \\
\hline Daily nap & -1.12 & 0.48 & 5.33 & 0.021 & $0.32(0.12,0.84$ \\
\hline Epworth Sleepiness Scale & -0.48 & 0.05 & 0.94 & 0.332 & $0.95(0.86,1.05$ \\
\hline \multicolumn{6}{|l|}{$28-32$ wk } \\
\hline Daily nap duration (min) & -0.12 & 0.00 & 5.88 & 0.015 & $0.98(0.97,0.99$ \\
\hline Petersburg Sleep Quality Index & -0.18 & 0.09 & 0.03 & 0.844 & $0.98(0.82,1.17$ \\
\hline Hosmer and Lemeshow test: $\chi^{2}=14.142$ & $d f=4$ & Sig $=0.007$ & & & \\
\hline \multicolumn{6}{|c|}{ Model summary: -2 log likelihood=192.774; Cox-Snell $R^{2}=0.006$; Nagelkerke $R^{2}=0.008$} \\
\hline Omnibus test of model coefficients: $\chi^{2}=0.833$ & $\mathrm{df}=1$ & Sig $=0.361$ & & & \\
\hline
\end{tabular}

$\mathrm{SE}$, standard error; OR, odds ratio; $\mathrm{Cl}$, confidence interval; df, degrees of freedom; Sig, significance. 
as the PSQI and the ESS score decreased, and decreased as daytime napping and nap duration increased; however, these associations were not significant. Most previous studies have examined the relationship of respiratory problems of pregnant women while sleeping with pregnancy outcomes, but few studies have been conducted on sleep quality during pregnancy. Some of those studies have assessed sleep quality in all trimesters of pregnancy $[20,28,33]$. Although those studies identified relationships between sleep quality and preterm birth, the results varied according to how each study defined the trimesters of pregnancy. In contrast, other studies examined sleep quality in just 1 trimester of pregnancy and found different results. In 2 studies, the relationship between sleep disorder and preterm birth was significant $[25,26]$. However, Dolatian et al. [29] did not report a significant relationship between sleep quality and preterm birth. Using different methods can play a role as a confounder. In most studies, sleep quality was assessed with the PSQI $[25,28,33]$, but the Insomnia Severity Index [29], the ESS [25,29], and the International Classification of Diseases, 9th revision, Clinical Modification codes [26] have also been used. Moreover, different inclusion criteria and sample sizes can contribute to differences in results.

The results of this study showed that according to the PSQI, the quality of sleep decreased as pregnancy progressed and a significant percentage of pregnant women had poor sleep quality. This finding has been confirmed in other studies [16,28,31]. Although Mindell et al. [17] did not report a significant difference in PSQI scores throughout pregnancy, they reported that sleep disorders increased as gestational age increased. The fact that most studies on sleep quality during pregnancy used the PSQI [34] improves the generalizability of the findings.

According to the results of the present study, a significant percentage of pregnant women had poor daytime sleep quality, but they showed an improvement in daytime sleep quality as gestational age increased and daytime napping and nap duration decreased. In contrast, Hutchison et al. [16] reported an increase in daytime napping as pregnancy progressed, but this result was not supported by other studies $[17,35]$. According to Sedov et al. [34], the effect of gestational age on sleep disorders during pregnancy has yet to be fully understood. The reason for differences among study results can be attributed to differences in gestational age at the time of data collection, the use of different methods to assess daytime sleep quality, and different modes of reporting. In addition, lifestyle differences throughout the world may be another contributing factor [16].

Furthermore, the present study found that an increase in daytime napping in the second trimester and in nap duration in the third trimester was associated with a reduced risk of cesarean section. Women suffering from poor sleep quality during pregnancy feel more tired and therefore have less energy to have a normal delivery [36]. Zhang et al. [20] reported that poor sleep quality in the first, second, and third trimesters of pregnancy were associated with an increased risk of cesarean section; however, this finding has not been reported in all studies $[29,37]$. Discrepancies among studies can be attributed to cultural and social differences, as well as differences in sleep definitions, sleep assessment tools, and gestational age at the time of sampling. None of the sleep variables was significantly related to birth weight, and likewise, other studies found no significant relationship between sleep quality and birth weight $[25,29]$. Given the relative lack of studies on this issue, more research is needed to reach a definitive conclusion; however, the relationships between sleep quality in pregnancy and the mode of delivery and birth weight were not the main focus of our study, so we did not investigate these variables in detail.

One of the strengths of this study is the sampling method. Qazvin, the setting of the research, is a city with a high proportion of immigrants due to its geographical and strategic status. Therefore, people from different social, economic, educational, and ethnic groups live together. Randomized cluster sampling from different areas of the city provided the opportunity to include participants from different socioeconomic classes in this study. Therefore, the authors believe that the results are generalizable to pregnant women in Iran. Using 2 questionnaires and questions about daily sleep disorders made this study different from previous studies. However, due to the limitations of the assessment, in this study, data were collected at 2 time periods, and no information was gathered on maternal sleep quality in the first trimester and before pregnancy. In addition, given the accuracy of polysomnography in assessing sleep status, it is recommended that this method be used alone or in combination with other methods in future studies. In this study, the relationship between sleep quality in pregnancy and preterm birth was not significant. Therefore, it is suggested that future studies should be conducted with larger samples of pregnant women.

A high percentage of pregnant women suffered from poor 
nighttime and daytime quality of sleep. Therefore, it is important to find these women and improve maternal sleep quality by simple advice and interventions (e.g., behavioral interventions). Poor sleep quality in the second and third trimesters of pregnancy was not associated with preterm birth or birth weight. However, increased daytime napping in the second trimester and nap duration in the third trimester showed associations with a lower risk of cesarean section, implying that improving sleep quality in pregnancy might reduce the rate of cesarean sections.

\section{CONFLICT OF INTEREST}

The authors have no conflicts of interest associated with the material presented in this paper.

\section{FUNDING}

None.

\section{ACKNOWLEDGEMENTS}

The authors would like to express their appreciation for the pregnant women who took part in this study.

\section{AUTHOR CONTRIBUTIONS}

Conceptualization: MM, FM. Data curation: FS, FR. Formal analysis: FM. Funding acquisition: None. Methodology: FM, $H H$, FS. Writing - original draft: FM, FR, FS. Writing - review \& editing: $\mathrm{FM}, \mathrm{MM}, \mathrm{HH}$.

\section{ORCID}

Farnaz Shaliha https://orcid.org/0000-0001-5579-8505 Maryam Mozaffari https://orcid.org/0000-0002-1194-9989 Faeze Ramezani https://orcid.org/0000-0001-6082-5889 Hamideh Hajnasiri https://orcid.org/0000-0003-2527-1504 Farnoosh Moafi https://orcid.org/0000-0002-3650-9293

\section{REFERENCES}

1. Manuck TA, Rice MM, Bailit JL, Grobman WA, Reddy UM, Wapner RJ, et al. Preterm neonatal morbidity and mortality by gestational age: a contemporary cohort. Am J Obstet Gynecol 2016;215(1):103.e1-103.e14.

2. Purisch SE, Gyamfi-Bannerman C. Epidemiology of preterm birth. Semin Perinatol 2017;41(7):387-391.

3. Martin JA, Hamilton BE, Osterman MJ, Driscoll AK, Drake P. Births: final data for 2017. Natl Vital Stat Rep 2018;67(8):1-50.

4. World Health Organization. Born too soon: the global action report on preterm birth; 2012 [cited $2020 \mathrm{Jul}$ 1]. Available from: https://www.who.int/pmnch/media/news/2012/201204_ borntoosoon-report.pdf.

5. Beck S, Wojdyla D, Say L, Betran AP, Merialdi M, Requejo JH, et al. The worldwide incidence of preterm birth: a systematic review of maternal mortality and morbidity. Bull World Health Organ 2010;88(1):31-38.

6. Sharifi N, Khazaeian S, Pakzad R, Fathnezhad Kazemi A, Chehreh $\mathrm{H}$. Investigating the prevalence of preterm birth in Iranian population: a systematic review and meta-analysis. J Caring Sci 2017;6(4):371-380.

7. de Freitas AS, Dobbler PC, Mai V, Procianoy RS, Silveira RC, Corso $A L$, et al. Defining microbial biomarkers for risk of preterm labor. Braz J Microbiol 2020;51:151-159.

8. Tellapragada C, Eshwara VK, Bhat P, Acharya S, Kamath A, Bhat $S$, et al. Risk factors for preterm birth and low birth weight among pregnant Indian women: a hospital-based prospective study. J Prev Med Public Health 2016;49(3):165-175.

9. Leal MD, Esteves-Pereira AP, Nakamura-Pereira M, Torres JA, Theme-Filha $M$, Domingues RM, et al. Prevalence and risk factors related to preterm birth in Brazil. Reprod Health 2016; 13(Suppl 3):127.

10. Shoja M, Shoja E, Gharaei M. Prevalence and affecting factors on preterm birth in pregnant women referred to Bentolhoda hospital-Bojnurd. J North Khorasan Univ Med Sci 2015;7(4): 855-863 (Persian).

11. Mehraban Z, Alizadeh L, Sadeghniyat HK. Surveying the relationship between sleep apnea and preterm birth in women referring to health centers of Ardabil. Urmia Med J 2016:26(11): 931-940 (Persian).

12. Gupta R, Dhyani M, Kendzerska T, Pandi-Perumal SR, BaHammam AS, Srivanitchapoom $P$, et al. Restless legs syndrome and pregnancy: prevalence, possible pathophysiological mechanisms and treatment. Acta Neurol Scand 2016;133(5):320-329.

13. Kocsis I, Szilágyi T, Turos J, Bakó A, Frigy A. Effect of a gymnastics program on sleep characteristics in pregnant women. Taiwan J Obstet Gynecol 2017;56(2):204-209.

14. Meharaban Z, Yahya S, Sadegniiat K. Restless legs syndrome during pregnancy and preterm birth in women referred to 
health centers of Ardabil. Iran Red Crescent Med J 2015;17(12): e24438.

15. Hung HM, Ko SH, Chen $\mathrm{CH}$. The association between prenatal sleep quality and obstetric outcome. J Nurs Res 2014;22(3): 147-154.

16. Hutchison BL, Stone PR, McCowan LM, Stewart AW, Thompson $J M$, Mitchell EA. A postal survey of maternal sleep in late pregnancy. BMC Pregnancy Childbirth 2012;12:144.

17. Mindell JA, Cook RA, Nikolovski J. Sleep patterns and sleep disturbances across pregnancy. Sleep Med 2015;16(4):483-488.

18. Abdullahzadeh M, Naji SA. The effect of matricaria chamomilla on sleep quality of elderly people admitted to nursing homes. Iran J Nurs 2014;27(89):69-79 (Persian).

19. Ram S, Seirawan H, Kumar SK, Clark GT. Prevalence and impact of sleep disorders and sleep habits in the United States. Sleep Breath 2010;14(1):63-70.

20. Zhang J, Li F, Lin Y, Sheng Q, Yu X, Zhang X. Subjective sleep quality in perimenopausal women and its related factors. J Nanjing Med Univ 2007;21(2):116-119.

21. Nodine PM, Matthews EE. Common sleep disorders: management strategies and pregnancy outcomes. J Midwifery Womens Health 2013;58(4):368-377.

22. Chang JJ, Pien GW, Duntley SP, Macones GA. Sleep deprivation during pregnancy and maternal and fetal outcomes: is there a relationship? Sleep Med Rev 2010;14(2):107-114.

23. Gunduz S, Kosger H, Aldemir S, Akcal B, Tevrizci H, Hizli D, et al. Sleep deprivation in the last trimester of pregnancy and inadequate vitamin D: is there a relationship? J Chin Med Assoc 2016;79(1):34-38.

24. Malekzadegan A, Moradkhani M, Ashayeri H, Haghani H. Effect of relaxation on insomnia during third trimester among pregnant women. Iran J Nurs 2010;23(64):52-58 (Persian).

25. Najar S, Sharafi F, Afshari P, Haghighizadeh MH. The relationship between sleep disorders during pregnancy and premature labor and low birth weight. Iran J Obstet Gynecol Infertil 2017;20(7):44-49 (Persian).

26. Felder JN, Baer RJ, Rand L, Jelliffe-Pawlowski LL, Prather AA. Sleep disorder diagnosis during pregnancy and risk of pre- term birth. Obstet Gynecol 2017;130(3):573-581.

27. Li R, Zhang J, Zhou R, Liu J, Dai Z, Liu D, et al. Sleep disturbances during pregnancy are associated with cesarean delivery and preterm birth. J Matern Fetal Neonatal Med 2017;30(6): 733-738.

28. Ota H, Hasegawa J, Sekizawa A. Effect of sleep disorders on threatened premature delivery. J Perinat Med 2017;45(1):57-61.

29. Dolatian M, Mehraban Z, Sadeghniat K. The effect of impaired sleep on preterm labour. West Indian Med J 2014;63(1):62-67.

30. Tomfohr-Madsen L, Cameron EE, Dunkel Schetter C, Campbell T, O'Beirne M, Letourneau N, et al. Pregnancy anxiety and preterm birth: the moderating role of sleep. Health Psychol 2019; 38(11):1025-1035.

31. Seyedahmadinejad FS, Golmakani N, Asgharipour N, Shakeri MT. Investigation of sleep quality during the third trimester of pregnancy and some related factors in primigravida women referred to health care centers in Mashhad-2014. Avicenna J Nurs Midwifery Care 2014;22(4):53-60 (Persian).

32. Afkham Ebrahimi A, Bandi G, Salehi M, Tafti K, Vakili Y, Farsi A. Sleep parameters and the factors affecting the quality of sleep in patients attending selected clinics of Rasoul-e-Akram hospital. Razi J Med Sci 2008;15:31-38 (Persian).

33. Okun ML, Luther JF, Wisniewski SR, Wisner KL. Disturbed sleep and inflammatory cytokines in depressed and nondepressed pregnant women: an exploratory analysis of pregnancy outcomes. Psychosom Med 2013;75(7):670-681.

34. Sedov ID, Cameron EE, Madigan S, Tomfohr-Madsen LM. Sleep quality during pregnancy: a meta-analysis. Sleep Med Rev 2018;38:168-176.

35. Loube DI, Poceta JS, Morales MC, Peacock MD, Mitler MM. Selfreported snoring in pregnancy. Association with fetal outcome. Chest 1996;109(4):885-889.

36. Beebe KR, Lee KA. Sleep disturbance in late pregnancy and early labor. J Perinat Neonatal Nurs 2007;21(2):103-108.

37. Tsai SY, Kuo LT, Lee CN, Lee YL, Landis CA. Reduced sleep duration and daytime naps in pregnant women in Taiwan. Nurs Res 2013;62(2):99-105. 